\title{
Association between dietary patterns and neuroimaging markers: a systematic review
}

Neuroimaging techniques can sensitively measure brain health and may elucidate potential diet-related mechanisms of cognitive ageing. Emerging evidence for a protective role of dietary patterns (DPs) on brain changes is inconsistent ${ }^{(1,2)}$. We are currently conducting a systematic review to improve our understanding of the relationship between DPs and brain ageing; in this abstract we present the studies reporting neuroimaging outcomes.

The review was registered with PROSPERO (CRD42020181423). EMBASE and MEDLINE databases were searched for studies published up to March 2020. Eligible studies for inclusion in the review were prospective studies in non-demented adults that examined whole DPs in relation to $\geq 1$ neuroimaging markers and published in the English language. Risk of bias was assessed using the Newcastle-Ottawa Scale (NOS).

From 9216 articles screened, 9 prospective studies involving 2540 participants from mid to older life met inclusion criteria: 7 assessed the Mediterranean dietary pattern (MeDi), 1 assessed adherence to the Alternative Healthy Eating Index (AHEI) and 1 used a-posteriori derived DPs. Eight studies examined the relationship between DPs and brain structure (e.g. grey matter[GMV]/ white matter[WMV]/hippocampal [HCV]/total brain volumes[TBV]/cortical thickness[CT]/white matter hyper intensities[WMH]/ infarcts and white matter microstructure integrity[WMI]. Three studies examined MeDi in relation to brain functional measures including beta-amyloid $(\mathrm{A} \beta)$ load $(\mathrm{N}=3)$ and brain glucose metabolism $(\mathrm{N}=2)$. Among the prospective studies assessing neuroimaging at 1 time point; MeDi was not associated with subsequent GMV, WMV, TBV or WMH $(\mathrm{N}=3)$. However, 2 studies found an association between higher MeDi adherence, decreased odds of cerebral infarcts 5.8 years later (OR: $0.89 ; 95 \%$ CI: $0.80,0.99)$ and improved WMI 8.9 years later. From studies assessing change in neuroimaging markers; MeDi was not associated with change in GMV or CT over 2-3 years $(\mathrm{N}=3)$, but higher $\mathrm{MeDi}$ adherence was associated with reduced TBV atrophy across 3 years $(\mathrm{N}=1)$. Two out of three studies found an association between lower MeDi adherence and increased A $\beta$ load across 2-3 years, and 2 found an association between lower MeDi adherence and increased rate of decline in brain glucose metabolism. From those examining a-posteriori DPs, one found an association between higher AHEI and greater HCV and one found a positive association between a prudent diet and greater HCV, but not with 4-year change in HCV. Given the heterogeneity of these data, meta-analysis was not performed. All studies were rated fair $(\mathrm{N}=7)$ or good $(\mathrm{N}=2)$ using NOS.

This review reveals conflicting evidence on the association between DPs and structural markers. Findings, whilst limited, were more consistent between MeDi and functional markers; this could be because functional changes may precede structural changes ${ }^{(3)}$. Future research should consider the use of multimodal neuroimaging and investigate DPs other than MeDi.

\section{References}

1. Mosconi L, Walters M, Sterling J, et al. (2018) BMJ Open (8), e019362.

2. Karstens A, Tussing-Humphreys L, Zhan L, et al. (2019) Am J Clin Nutr 109(2), 361-368.

3. Jack CR, Knopman DS, Jagust WJ, et al. (2010) The Lancet Neurol 9(1), 119. 